\title{
LA TIPIFICACIÓN SEXUAL DEL TRABAJO MERCANTIL. EL CASO VALENCIANO
}

\author{
Ma JOSÉ FRAU LLINARES \\ D. de C. Sociales y de la Educación. Universidad de Alicante.
}

\section{INTRODUCCIÓN}

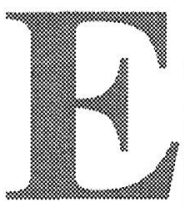

n las últimas décadas hemos asistido a cambios muy importantes en la estructura de la fuerza de trabajo en las sociedades industrializadas. Uno de esos cambios, sin duda, ha sido su feminización.

El desarrollo de las economías capitalistas en el período de postguerra creó las condiciones adecuadas para la incorporación de la mujer al mercado y, a partir de ese momento, su presencia en el trabajo remunerado se ha ido incrementado constantemente.

En España, también se ha producido en las dos últimas décadas un aumento considerable de la participación de las mujeres en la esfera mercantil, pero, a pesar de ello, la tasa de actividad femenina no ha alcanzado niveles comparables al resto de países europeos. Precisamente, el valor tan bajo que presenta la tasa de actividad de la población española, que se sitúa alrededor del $60 \%$ (respecto a la población entre 16 y 64 años), se explica generalmente por la escasa participación de las mujeres.

Cuando se inició la recesión económica en los años 70, en España se partía de niveles de actividad femenina mínimos, y la evolución experimentada durante este periodo, claramente positiva, no ha sido suficiente para igualarnos con el resto de los países de nuestro contexto. El resultado es que España sigue siendo uno de los países de la Unión Europea con la tasa de actividad femenina más baja, tan sólo la correspondiente a Irlanda presenta un valor inferior.

La tasa de actividad femenina en estos años ha seguido, al igual que en la mayoría de los países, una tendencia inversa a la tasa de actividad masculina, se ha incrementado, mientras que esta última descendía. Ahora bien, a pesar de este mayor dinamismo de las mujeres, las diferencias en las tasas de actividad siguen siendo importantes y se siguen manteniendo las desventajas comparativas que las mujeres han sopor- 
tado históricamente. La mayor participación femenina no ha supuesto la eliminación de la discriminación en el mercado laboral: las mujeres trabajan en condiciones más precarias que los hombres, lo hacen por menos salario y, además, aunque con matices, su empleo suele ser más vulnerable en períodos de recesión.

La idea de que el empleo de las mujeres se ve más afectado que el empleo de los hombres en los ciclos económicos, es decir, que las mujeres son las primeras en ser despedidas en períodos de recesión y contratadas en períodos de expansión cuando escasea la mano de obra masculina, ha sido analizada ${ }^{\prime}$ a la luz del comportamiento del mercado de trabajo en la etapa de recesión iniciada en la década de los 70 .

La recesión mundial y la consiguiente crisis de empleo que trajo consigo hizo pensar que la tendencia iniciada en los años 50 y 60 se iba a invertir, que las mujeres iban a ver disminuidas sus cuotas de participación en la ocupación volviendo de nuevo a sus hogares. Sin embargo, no parece demostrada la hipótesis de que las mujeres actúan de amortiguador frente a las fluctuaciones del empleo, puesto que han seguido incrementando sus tasas de participación laboral a pesar de las altas tasas de paro masculinas.

Hay cierta coincidencia en que las mujeres han estado relativamente protegidas ante las fluctuaciones del empleo en esta etapa de recesión, lo que se explica desde diferentes perspectivas.

Uno de los argumentos desarrollados apunta a que la segregación ocupacional, sobradamente constatada, ha desempeñado un papel fundamental en esta protección. La tendencia secular de las mujeres hacia el empleo en el sector servicios, en un periodo en que se ha producido una reestructuración ocupacional consistente en una terciarización del empleo y una disminución en el empleo industrial, tradicionalmente masculino, explicaría que haya disminuido la vulnerabilidad del empleo femenino en su conjunto.

Así, el mejor comportamiento de los sectores en los que se concentra la fuerza de trabajo femenina la ha hecho más resistente ante las fluctuaciones vividas, aunque es probable que, en sectores determinados, las mujeres hayan actuado de amortiguador al ocupar los puestos de trabajo más inestables.

Jill Rubery señala que «no hay evidencia que sugiera que las mujeres universalmente asumen el papel de amortiguador en el sistema de mercado de trabajo asalariado. $»^{2}$ Aunque las mujeres hayan podido actuar de reserva flexible de mano de obra en algunos sectores consi-

- En Jil Rubery (comp.): Las mujeres y la recesión, Ministerio de Trabajo y Seguridad Social, Madrid, 1993, se analiza el comportamiento en Estados Unidos, Francia, Italia, Gran Bretaกัa y España.

2 Jil Rubery: op.cit. p. 398. 
derados individualmente, la segregación ocupacional habría actuado en ese período de escudo protector del empleo femenino total.

Otro de los argumentos expuestos apunta al menor coste de la fuerza de trabajo femenina como explicación del mantenimiento de su participación en la actividad económica en la recesión. Su menor coste y su mayor flexibilidad resultan un atractivo para el empresariado, convirtiéndose, por esta razón, la fuerza de trabajo femenina en sustituta de la masculina en momentos en que se persigue como objetivo prioritario abaratar los costes de producción.

En este argumento, al intentar explicar las causas del mantenimiento de los niveles de empleo femenino, se aborda una cuestión que resulta imprescindible plantear cuando se trata la desigualdad sexual en el trabajo: el menor coste y la mayor flexibilidad de la fuerza de trabajo femenina, lo que representa una clara desventaja relativa de las mujeres.

Estas características de la fuerza de trabajo femenina - más barata y más flexible- se explican generalmente a partir de la teoría del mercado dual, según la cual las mujeres, junto a los jóvenes y los inmigrantes, formarían parte del llamado mercado secundario.

La idea básica de esta teoría parte de la concepción de que no hay un único marco de contratación. El mercado está dividido en dos segmentos diferenciados y sin apenas movilidad entre ellos, denominados sectores primario y secundario. «El primero ofece puestos de trabajo con salarios relativamente elevados, buenas condiciones de trabajo, posibilidades de avance, equidad y procedimientos establecidos en cuanto a la administración de las normas laborales y, por encima de todo, estabilidad de empleo». En el sector primario se diferencia entre un segmentos superior -formado por trabajos profesionales y directivosy un segmento inferior. «En cambio, los puestos del sector secundario tienden a estar peor pagados, a tener condiciones de trabajo peores y pocas posibilidades de avance; a tener una relación muy personalizada entre los trabajadores y los supervisores que deja un amplio margen para el favoritismo y lleva a una disciplina laboral dura y caprichosa; $y$ a estar caracterizados por una considerable inestabilidad de empleo y una elevada rotación de la población trabajadora». ${ }^{3}$

Con esta diferenciación, la teoría de la segmentación rompe con la neutralidad del mercado de trabajo: la existencia de buenos y malos trabajos no deriva de la mayor o menor especialización de los trabajadores, más bien viene determinada por la propia estructura industrial.

Ahora bien, una vez establecidos los segmentos del mercado, la cuestión está en cuáles son los mecanismos que adscriben a los diferentes grupos sociales a cada uno de ellos. Para los teóricos de la

3 M.Piore: «Notas para una teoría de la estratificación del mercado de trabajo», en L. Toharia (comp.) : El mercado de trabajo: Teorías y aplicaciones. Alianza ed., Madrid, 1983. 
dualización, no es el mercado el que realiza esa adscripción, sino, más bien, ciertos rasgos socioculturales de los propios grupos sociales que constituyen cada uno de los segmentos.

Hay grupos sociales que, por tener tener otros intereses ajenos al mercado y un compromiso más débil con él, están dispuestos en mayor medida a aceptar las condiciones de trabajo del mercado secundario.

Entre estos grupos, según M.J. Piore, se encuentran las mujeres casadas, los trabajadores negros, los jóvenes, los inmigrantes. «El sistema capitalista encuentra estas clases y no las crea. (...) Los emigrantes (extranjeros e interiores), los trabajadores y las mujeres son atractivos precisamente porque pertenecen a otra estructura socioeconómica y consideran el empleo industrial como un aditamento de sus papeles primarios. Están dispuestos a aceptar trabajos temporales porque ven su compromiso con estos trabajos como algo temporal y son capaces de soportar el cambio y la incertidumbre de la economía industrial porque tienen actividades económicas tradicionales a las que pueden volver.» ${ }^{4}$

Aún aceptando la descripción básica de los segmentos del mercado, estudiosos del trabajo femenino han cuestionado la imagen de las mujeres como trabajadoras del sector secundario. ${ }^{5}$ El trabajo de las mujeres presenta un mayor grado de inestabilidad y difícilmente ocupa los puestos de dirección y planificación (lo que constituiría el segmento primario superior), pero muchos de los sectores en los que se concentran las mujeres no sólo no presentan las características del mercado secundario, sino más bien exigen una formación continuada y una alta cualificación y experiencia. Y es más, en muchas de estas profesiones las mujeres son fuerza de trabajo preferente más que sustitutoria. Se señala, además, que las mujeres recurren aún, en mayor medida que los hombres, a la formación para aumentar sus oportunidades de acceder al mercado laboral, lo que entraría en contradicción con la falta de interés en permanecer en él.

4 Piore, M. «El dualismo como respuesta al cambio y a la incertidumbre», en Toharia, L. (comp.) «El mercado de trabajo: Teorías y aplicaciones». Ed. Alianza, 1983. Madrid. Pgn. 254.

5 Algunas de estas críticas pueden verse en los artículos de B. Beehey «Género y Trabajo. Replanteamiento de la definición de trabajo» y de F. Bettio «Segregación y debilidad. Hipótesis altgernativas en el análisis del mercado de trabajo», en C. Borderías, C. Carrasco y C. Alemany (comp.), Las mujeres y el trabajo, Icaria, Barcelona, 1994. 


\section{LOS DATOS EMPÍRICOS}

\section{El trabajo femenino en la Comunidad Valenciana}

La creciente participación de la mujer en el empleo, a la que me he referido, queda patente también en la Comunidad Valenciana.

TABLA 1: EVOLUCIÓN DE LA TASA DE FEMINIZACIÓN DE LA OCUPACIÓN Y EL PARO. COMUNIDAD VALENCIANA

\begin{tabular}{|l|c|c|c|c|}
\hline TASA FEMINIZACIÓN & $\mathbf{1 9 7 6}$ & $\mathbf{1 9 8 5}$ & $\mathbf{1 9 9 0}$ & $\mathbf{1 9 9 4}$ \\
\hline Ocupación & 28,8 & 29,4 & 33,7 & 33,8 \\
\hline Paro & 43,8 & 35,8 & 51,2 & 50 \\
\hline & \multicolumn{4}{|c|}{ Per. recesión Per. recuperac. Per. recesión } \\
\cline { 2 - 5 }
\end{tabular}

FUENTE: Encuesta de Población Activa. INE.

Los datos contenidos en la tabla 1 nos ilustran sobre la feminización de la actividad laboral en la comunidad. Feminización que resulta tanto del aumento de mujeres con un puesto de trabajo como de aquellas otras que pretenden acceder a uno sin conseguirlo y que pasan a engrosar las filas del paro. Tanto unas como otras constituyen colectivos que se han ampliado en el período considerado.

Así, la etapa comprendida entre 1976-1994 ha supuesto para las mujeres la creación de 46,3 mil empleos netos según la Encuesta de Población Activa, lo que contrasta con la tendencia de la ocupación de los hombres, colectivo que ha perdido en ese mismo período 89 mil empleos. El resultado de todo ello ha sido un aumento de la participación femenina en la ocupación, lo que ha supuesto que la tasa de feminización se haya incrementado del $28,8 \%$ al $33,8 \%$.

El incremento de la ocupación femenina no ha sido suficiente para dar respuesta al amplio número de mujeres que en estos años han decidido integrarse en la población activa, por lo se ha producido también una feminización del desempleo. En 1993 hay aproximadamente -según la E.P.A.- 177,7 mil paradas más que en 1976. El desempleo de las mujeres, al igual que ocurre con la ocupación, ha crecido a un ritmo superior a la media, pasando de representar el $43,8 \%$ de los parados en 1976 a representar el 50\% en 1994. 
El comportamiento de las tasas de feminización, tanto de la ocupación como del paro, no ha sido homogénea durante todo la etapa.

En el período 1976-1985 se produce una caída de la ocupación total en todos los sectores excepto en los servicios - «cuya tasa de crecimiento anual es de 0,8 entre $1975-80$ y 1,9 entre $1980-85{ }^{6}{ }^{6}$-, sector en el que se concentran especialmente las mujeres. La caída de la ocupación afecta tanto a hombres como a mujeres, permaneciendo la participación femenina relativamente estable. En paralelo, se produce una caída de la participación femenina en el desempleo, efecto, posiblemente, del «paro desanimado» que afectaba especialmente a las mujeres en períodos de recesión.

El mayor incremento de la tasa de feminización se produce en el período de recuperación del empleo (1985-90), con gran diferencia respecto a los demás períodos. En esta etapa se incrementa el empleo en todos los sectores, excepto en la agricultura que sigue presentando valores negativos. En el sector servicios se eleva la tasa de crecimiento anual al 4,3. Pero es, también, el período en que la feminización del desempleo adquiere mayor relevancia. En ese período, en el que acuden al mercado laboral las llamadas «paradas desanimadas» ante las buenas expectativas, la tasa de feminización de desempleo supera el $50 \%$.

Por último, hay que señalar que la tendencia del nuevo ciclo que se inicia a principios de los 90 , caracterizado de nuevo por la pérdida de empleo, repercute en la participación femenina en la ocupación, que vuelve a estabilizarse como ocurrió en el período 76-85. La participación femenina en el desempleo, sin embargo, en este nuevo ciclo recesivo, no nos muestra tan claramente los efectos del paro desanimado, pues presenta una caída, pero muy tenue.

Todo esto nos indica que el empleo femenino ha sido especialmente sensible a los ciclos económicos. Ha sido en el período de recuperación en el que más ha aumentado la tasa de feminización. Ahora bien, también hay que destacar que, en ningún momento, la tasa de feminización retrocede -en los períodos de recesión, aunque mínimamente, también se incrementa su valor-, lo que nos permite considerarla como parte del segmento permanente de la fuerza de trabajo.

\section{La segregación ocupacional}

La progresiva incorporación de la mujer al mercado laboral, no se ha traducido en una diversificación de los empleos femeninos. El mercado de trabajo sigue caracterizándose por un alto grado de segrega-

" Datos tomados de: C.Hernández Pacual, Regulación, flexibilidad y segmentación de los mercados de trabajo, Instituto de Cultura Juan Gil Albert, Alicante, 1995. p. 45. 
ción sexual, entendiendo por segregación, tal como señala la OCDE, «la existencia de un fuerte desequilibrio en la distribución de las personas entre las distintas actividades u ocupaciones económicas, en función de alguna característica personal, como puede ser el sexo o la raza».

La mayoría de las mujeres se concentran en algunas ramas de actividad y en un reducido número de profesiones que ya se consideran feminizadas, dando lugar a lo que conocemos como segregación horizontal. Pero, además, suelen situarse en los niveles socio-profesionales bajos o intermedios, con pocas oportunidades de ocupar puestos de dirección. De esta manera, la segregación se manifiesta en su doble vertiente: «horizontal» (concentración de los empleos femeninos en un pequeño número de sectores de actividad y de profesiones) y «vertical» (concentración de los empleos femeninos en las categorías que se encuentran situadas en la parte baja de la jerarquía profesional). ${ }^{7}$

\section{a. Sectores económicos y ramas de actividad}

La tabla 2, en la que aparecen los datos referidos a la distribución de la población ocupada por sectores económicos, nos muestra la profunda división sexual del trabajo existente en la Comunidad Valenciana y su comparación con el conjunto nacional.

En la Comunidad Valenciana, al igual que ocurre en el conjunto español, hombres y mujeres tienen una mayor o menor presencia en los diferentes sectores conómicos. Más de las tres cuartas partes de las mujeres ocupadas trabajan en el sector servicios, en el cual están sobrerepresentadas (IRF: 1,3). En la industria, sector que absorbe al $18,9 \%$ de las mujeres ocupadas (cuatro puntos por encima del conjunto español), están infrarrepresentadas, al igual que ocurre en la agricultura (IRF: 0,7). En este último sector, el porcentaje de mujeres ocupadas es mínimo, así como su IRF de 0,4 , que se sitúa por debajo del correspondiente a la media nacional. Hay que señalar, también, la escasa o nula presencia de las mujeres en la construcción.

El hecho de que más de las tres cuartas partes de las mujeres con empleo se concentren en los servicios, sector que como hemos visto es el que mejor comportamiento ha tenido en todo el período, puede ser una de las razones del mayor crecimiento relativo de la ocupación femenina. La segregación ocupacional ha podido estar desempeñando esa función protectora para el empleo femenino a la que me refería anteriormente. 
TABLA 2: DISTRIBUCIÓN PORCENTUAL DE LA POBLACIÓN OCUPADA, SEGÚN SECTORES ECONÓMICOS Y SEXO. COMUNIDAD VALENCIANA.

\begin{tabular}{|l|c|c|c|c|c|c|}
\cline { 2 - 7 } \multicolumn{1}{c|}{} & \multicolumn{4}{c|}{ Comunidad Valenciana } & \multicolumn{2}{c|}{ Nacional } \\
\hline SECTORES & $\begin{array}{c}\text { varones } \\
\%\end{array}$ & $\begin{array}{c}\text { mujeres } \\
\%\end{array}$ & $\begin{array}{c}\text { tasa de } \\
\text { feminiz }\end{array}$ & I.R.F. & $\begin{array}{c}\text { Tasa de } \\
\text { feminiz }\end{array}$ & I.R.F. \\
\hline Agricultura & 9,6 & 3,3 & 14,5 & 0,4 & 23,8 & 0,7 \\
\hline Industria & 30,3 & 18,9 & 23,4 & 0,7 & 22,5 & 0,7 \\
\hline Construcción & 13,5 & 0,8 & 2,8 & - & 2,9 & - \\
\hline Servicios & 46,5 & 76,9 & 44,6 & 1,3 & 42,5 & 1,3 \\
\hline Total \\
(en miles)
\end{tabular}

FUENTE: Encuesta sociodemográfica (1991, INE) y elaboración propia.

La participación femenina en el conjunto del empleo disminuye con la edad; la tasa de feminización, como vemos en la tabla 3 , sigue una tendencia inversa a la edad, presentando los grupos más jóvenes

TABLA 3: TASA DE FEMINIZACIÓN DEL EMPLEO, SEGÚN SECTORES ECONÓMICOS Y GRUPOS DE EDAD. COMUNIDAD VALENCIANA.

\begin{tabular}{|l|c|c|c|c|c|c|c|c|}
\cline { 2 - 10 } \multicolumn{1}{c|}{} & \multicolumn{2}{c|}{$\begin{array}{c}\text { Menos de } 30 \\
\text { años }\end{array}$} & \multicolumn{2}{c|}{$\begin{array}{c}\text { Entre } 30 \text { y } 39 \\
\text { años }\end{array}$} & \multicolumn{2}{|c|}{$\begin{array}{c}\text { Entre } 40 \text { y } 49 \\
\text { años }\end{array}$} & \multicolumn{2}{|c|}{50 años y más } \\
\hline SECTORES & T.F. & IRF & T.F. & IRF & T.F. & IRF & T.F. & IRF \\
\hline Agricultura & 12,8 & 0,3 & 18,8 & 0,5 & 22,6 & 0,7 & 10,7 & 0,4 \\
\hline Industria & 24,4 & 0,6 & 20,5 & 0,6 & 9,9 & 0,3 & 8,9 & 0,3 \\
\hline Construcción & 3,4 & 0,8 & 1,9 & - & 2,4 & - & 1 & - \\
\hline Servicios & 64,3 & 1,5 & 53,6 & 1,5 & 52,9 & 1,8 & 47,7 & 2,0 \\
\hline & 39,3 & & 34,2 & & 29,5 & & 23,9 & \\
\hline
\end{tabular}

FUENTE: Encuesta sociodemográfica (1991, INE) y elaboración propia. 
TABLA 4: DISTRIBUCIÓN DE LA POBLACIÓN OCUPADA SEGÚN LA SITUACIÓN PROFESIONAL Y SEXO (\%). COMUNIDAD VALENCIANA.

\begin{tabular}{|l|c|c|c|c|}
\hline SITUACIÓN PROFESIONAL & varones & mujeres & T. femin. & IRF \\
\hline Empresario con asalariados & 6,2 & 2 & 13,7 & 0,4 \\
\hline Empresario sin asalariados & 15 & 10,9 & 23,6 & 0,7 \\
- con ayuda familiar & 4,8 & 3,6 & 23,2 & 0,7 \\
- trab. por cuenta propia & 10,2 & 7,3 & 23,8 & 0,7 \\
\hline Miembro de cooperativa & 1,8 & 0,4 & 13,1 & 0,4 \\
\hline Ayuda familiar & 2,7 & 5,7 & 53,8 & 1,6 \\
• convive con empres. & 1,8 & 4,1 & 58,4 & 1,8 \\
- no convive con emp. & 0,9 & 1,6 & 45,4 & 1,4 \\
\hline Asalariado emp. privada & 66,3 & 55,8 & 29,3 & 0,9 \\
\hline Asalariado Admón. Púb. & 8,1 & 18,8 & 52,8 & 1,6 \\
\hline Asalar. hogares famil. & 0 & 6,4 & 99 & 2,9 \\
\hline Total & 100 & 100 & 32,8 & \\
\hline (en miles) & 886,5 & 432,6 & & \\
\hline
\end{tabular}

FUENTE: Encuesta sociodemográfica 1991. INE.

las mayores tasas de participación laboral. Sin embargo, y en contra de lo que se podría esperar, la segregación por sectores económicos se da en todos los grupos, incluso entre las más jóvenes. Aunque algo suavizado (la segregación sexual en industria y servicicios, sectores que engloban la mayor parte del empleo, es más extrema en los grupos de más edad), el grupo de mujeres más joven sigue teniendo una clara sobrerepresentación en el sector servicios, mientras que en la industria se mantiene por debajo del nivel de equilibrio.

$\mathrm{Al}$ desagregar los sectores económicos por ramas de actividad (tabla 5), observamos cómo la ocupación femenina se concentra en un número muy reducido de éstas. Se localiza fundamentalmente en cinco de las trece ramas de actividad consideradas. Estas ramas son: comercio (25\%), industrias manufactureras $(15,3)$, sanidad y asistencia social $(10,5)$, otros servicios $(10,3)$ y educación $(9,8)$. Estos cinco subsectores, en conjunto, absorben al $71 \%$ de las mujeres ocupadas. 
TABLA 5: DISTRIBUCIÓN DE LA POBLACION OCUPADA POR RAMAS DE ACTIVIDAD, SEGÚN SEXO (\%). COMUNIDAD VALENCIANA.

\begin{tabular}{|l|c|c|c|c|}
\hline RAMA DE ACTIVIDAD & varones & mujeres & t. feminiz. & IRF \\
\hline Agricultura, ganadería y pesca & 9,6 & 3,3 & 14,5 & 0,4 \\
\hline Industrias extractivas & 0,4 & 0 & - & - \\
\hline Energía e industrias básicas & 7 & 2,2 & 13,2 & 0,4 \\
\hline Industria metálica y eléctrica & 5,9 & 1,4 & 10,7 & 0,3 \\
\hline Industrias manufactureras & 17 & 15,3 & 30,5 & 0,9 \\
\hline Construcción & 13,5 & 0,8 & 2,8 & - \\
\hline Comercio & 16,9 & 25 & 41,9 & 1,3 \\
\hline Hostelería & 4,3 & 5,7 & 39,2 & 1,2 \\
\hline Transportes y comunicaciones & 6,8 & 2,2 & 13,4 & 0,4 \\
\hline Instituciones financieras & 3,2 & 2 & 23,2 & 0,7 \\
\hline Administraciones públicas & 4,3 & 5 & 36 & 1,1 \\
\hline Educación & 2,6 & 9,8 & 65 & 2 \\
\hline Sanidad y asistencia social & 2,4 & 10,5 & 67,7 & 2 \\
\hline Otros servicios & 6 & 10,3 & 45,5 & 1,4 \\
\hline Servicio doméstico & 0 & 6,4 & 99,3 & 3,0 \\
\hline Total & 100 & 100 & 32,8 & \\
\hline en miles) & 886,5 & 432,6 & & \\
\hline
\end{tabular}

FUENTE: Encuesta sociodemográfica (1991, INE) y elaboración propia.

Con menos peso cuantitativo, pero con gran significación por la tasa de feminización que presenta, hay que destacar el «servicio doméstico», que engloba al 6,4\% de las ocupadas, siendo además el subsector en el que podemos suponer que hay más empleo encubierto, por lo que la cifra está, sin duda, muy infravalorada. Es muy significativo que prácticamente todo el empleo en este subsector es femenino.

Las mujeres están sobrerrepresentadas en el «servicio doméstico» (IRF: 3), «educación» (IRF: 2), «sanidad y asistencia social» (IRF: 2), «otros servicios» (IRF: 1,4), «comercio» (IRF: 1,3), «hostelería» (IRF: 1,2) y «administraciones públicas» (IRF: 1,1). En los tres primeros, además, la presencia de las mujeres es igual o superior a la de los hombres, lo que nos permite hablar de ocupaciones feminizadas.

Por otra parte, están claramente subrrepresentadas en los subsectores industriales - excepción hecha de «industrias manufactureras» en las que el IRF alcanza un valor cercano a la unidad-, en la agricultura y en la construcción y dentro del sector servicios, en el subsector de «transportes y comunicaciones». 


\section{b. Situación profesional}

Otra de las características de la estructura ocupacional que nos ilustra sobre la tipificación sexual del trabajo es la situación profesional, variable que define la posición de las personas respecto a su relación laboral. En ella podemos distinguir, en primer lugar, a aquéllos que realizan el trabajo por cuenta propia de aquellos otros que son asalariados. En el primer grupo, incluimos las categorías de empleador, empresario sin asalariados, ayuda familiar y cooperativista, mientras que en el segundo grupo es interesante diferenciar entre los asalariados del sector privado y los del sector público.

En este sentido, los dos grandes rasgos que caracterizan al empleo femenino en la Comunidad Valenciana son su mayor grado de asalarización, así como un mayor nivel de adscripción al sector público.

La tasa de asalarización de las mujeres es del $81,1 \%$ frente al 74,4\% correspondiente a los varones (tabla 4). Consideradas por separado las diferentes categorías que integran este grupo, observamos que la mayor tasa de participación de las mujeres se da en el sector público. El predominio de la mujer en este sector es claro, superando el nivel de paridad entre los sexos.

Entre los asalariados del sector privado, sin embargo, observamos que la participación femenina desciende, situándose por debajo su media de ocupación, lo que se traduce en un IRF que, aunque cercano a la unidad $(0,9)$, se mantiene por debajo de ésta. Por último, señalar a los asalariados de los hogares familiares que en un $98 \%$ son mujeres y representan el 6,4\% del empleo femenino.

En el resto de las situaciones profesionales la participación femenina se sitúa por debajo de su media, excepto en el caso de la «ayuda familiar», que está fuertemente feminizada. Esta figura, frontera, como señala José Ignacio Casas, entre los roles familiares y los roles laborales, en la que «el beneficio y el resultado público del trabajo realizado es controlado, gestionado y recibido su consentimiento por el marido, titular de la explotación ${ }^{8}$, sigue integrando al 5,7\% de las mujeres ocupadas.

En resumen, el empleo femenino en la Comunidad Valenciana aparece claramente localizado en el sector «servicios»y, especialmente, en los subsectores de «comercio», «industrias manufactureras», «educación», «sanidad» y «otros servicios».

Tres de estos subsectores, en los que se ha clasificado la ocupación, pueden considerarse claramente feminizados: «educación», «sanidad» y «servicio doméstico», al superar su participación el nivel de paridad entre los sexos. Igualmente, hay que destacar la mayor tendencia de

\& J.I. Casas. «Características del trabajo de la mujer: el caso español». Sociología de trabajo $n^{\circ}$ 3. Ed. Siglo XXI. Madrid, 1988. Pág. 28 
las mujeres al trabajo asalariado y su escasísima presencia entre los empleadores, así como su adscripción al sector publico, categoría en la que también se supera el nivel de paridad.

Respecto a esto último, suelen destacarse diversos rasgos que hacen al sector público especialmente atractivo para las mujeres. Por una parte, se destacan sus horarios intensivos y claramente fijados que lo hacen más compatible que el sector privado con el trabajo de reproducción. Esto, sin embargo, es aplicable a la «administración» y «educación», pero no a la «sanidad», subsector en el que también está sobrerrepresentada la mujer. Por otra parte, se destaca que este sector ofrece más seguridad en el empleo -rasgo interesante para las mujeres que como sabemos suelen ser un colectivo más vúlnerables a la fluctuación del empleo-, y, especialmente, el hecho de que el sector público, por su criterios de selección, es menos discriminatorio para la mujer en la contratación.

Esta mayor presencia de las mujeres en ciertas actividades, la mayoría de ellas ligadas a la educación, sanidad o asistencia social, deriva tanto del comportamiento de la demanda como de la oferta. El hecho de que el empleo que más ha crecido en el periodo en que la mujer se ha incorporado al mercado de trabajo se haya creado en ese ámbito, o que el sector público discrimine menos, por sus formas de contratación, a la hora de seleccionar a hombres y mujeres, explica, en parte, esa especialización.

Pero también hay que tener presente las diferentes elecciones que hacen los hombres y mujeres. Estas últimas, a menudo, ante la falta de una cualificación formal, tienen interés y necesidad de aprovechar esa cualificación no oficial, generalmente no reconocida como tal, que han adquirido mediante su adiestramiento y experiencia en la esfera reproductiva, y que se adapta a muchas de las profesiones -en las que no se exige una cualificación oficial-que son una especie de continuidad de aquellas tareas propias del ámbito doméstico para las que la mujer ha sido socializada y a las que dedican gran parte de su tiempo diario. También, a menudo, se da una falta de motivación por parte de las mujeres para dirigirse hacia sectores ocupacionales concretos, porque en ellos encuentran fuertes resistencias para integrarse en las redes de relaciones, no sólo laborales sino también personales y culturales, propias de las profesiones muy masculinizadas. Todos estos elementos son, entre otros, rasgos derivados tanto de la oferta como de la demanda de fuerza de trabajo que nos ayudan a entender la especializacion sexual de las ocupaciones.

Ahora bien, esta división sexual del trabajo, en sí misma, no tendría porqué ser jerárquica. La especialización en ciertos sectores económicos no implica que las mujeres tengan que ocupar una posición inferior a la de los hombres. Sin embargo, la realidad nos muestra que, 
además de la segregación horizontal, se produce una jerarquización vertical de las ocupaciones, aún en aquellas claramente feminizadas, evidenciándose la ausencia de las mujeres en las categorías laborales más elevadas

\section{c. Las categorías laborales}

Si atendemos a la distribución de la población ocupada según las categorías laborales, observamos que las mujeres tienen una escasa presencia en las categorías más altas.

Los datos contenidos en la tabla 6, en la que las categorías laborales -concepto que constituye un reconocimiento de la experiencia y la especialización, y que suele determinar el nivel de remuneración- aparecen ordenadas de mayor a menor nivel, son suficientemente reveladores de la jerarquización sexual del trabajo. La tasa de feminización alcanza los valores más elevados en la parte baja de la escala de cada uno de los subgrupos.

Considerando el índice de representación femenina de cada categoria en relación a la participación en el empleo total (IRF (1)) se observa esta gradación, aunque los valores quedan algo distorsionados por la diferente proporción del empleo femenino o masculino en cada uno de los subsectores considerados (escala técnica, de producción o de servicios). Por ello, resulta más clarificador, para el objetivo que se persigue, relacionar la mayor o menor participación de las mujeres en cada una de las categorías laborales, en relación a la importancia relativa del empleo femenino en el subgrupo del cual dicha categoría laboral forma parte.

Con este objeto, se ha elaborado el segundo Indice de Representación Femenina (IRF (2)), a la vista del cual se confirma claramente la tesis mantenida. En todos los subgrupos, el IRF presenta una gradación que va de los valores más bajos en la parte alta de la escala a los valores más altos en la parte baja de ésta. Hay que señalar una excepción: la categoría de «técnicos sin titulación» oficial, en la que no sorprende que la presencia de las mujeres sea menos significativa.

Ahora bien, esta jerarquización no alcanza el mismo nivel en cada uno de los subgrupos establecidos. Aquéllos en los que la tasa de participación femenina es mayor, como son los subgrupos de «personal de servicios»y «personal técnico», la jerarquización sexual está algo más atenuada.

Otro indicador cuyos valores confirman también la existencia de segregación vertical del trabajo es el nivel de responsabilidad que sobre el personal tienen los hombres y las mujeres. 
TABLA 6: POBLACIÓN OCUPADA SEGÚN CATEGORÍA PROFESIONAL Y SEXO

\begin{tabular}{|l|c|c|c|c|c|}
\hline CATEGORÍA LABORAL & varones & mujeres & t. femin. & IRF(1) & IRF(2) \\
\hline - Directores & 1,4 & 0,3 & 11 & 0,3 & \\
\hline - Escala pers. técnico & & & & & \\
Titul. superior & 3,5 & 4,7 & 42,8 & 1,2 & 0,8 \\
Titul. medio & 3,8 & 9,8 & 60,6 & 1,7 & 1,1 \\
Técnic. sin titulac. & 0,8 & 0,2 & 14,2 & 0,4 & 0,3 \\
Ayud. técnico & 0,4 & 1,7 & 71,4 & 2 & 1,3 \\
Auxiliar técnico & 0,5 & 2,8 & 77 & 2,9 & 1,5 \\
\hline - Esc. pers. administ. & & & & & \\
Jefe administrativo & 3,3 & 1,1 & 18 & 0,5 & 0,4 \\
Oficial administrativo & 4,4 & 4,3 & 33,9 & 0,9 & 0,7 \\
Auxiliar administrativo & 4,6 & 15 & 63,6 & 1,8 & 1,4 \\
Aspirante, aprendiz & - & 0,3 & 80 & 2,2 & 1,7 \\
\hline Esc. per. producción & & & & & \\
Jefe de taller & 1,1 & 0,1 & 4,3 & 0,1 & 0,2 \\
Encarg. capat. jefe & 3,6 & 0,1 & 2,2 & 0,1 & 0,1 \\
Oficial & 20,8 & 6,7 & 14,3 & 0,4 & 0,7 \\
Peón especialista & 10 & 7,4 & 27 & 0,7 & 1,3 \\
Peón & 16,5 & 11,6 & 27,3 & 0,7 & 1,3 \\
Aprendiz & 2,2 & 1,6 & 29,3 & 0,8 & 1,4 \\
\hline Escala per. servicios & & & & & \\
Oficial & 2,1 & 2,9 & 43,3 & 1,2 & 0,7 \\
Mozo-pinche & 1 & 1,7 & 57,5 & 1,6 & 1 \\
Subalterno & 1,7 & 8,7 & 69,6 & 1,9 & 1,1 \\
\hline - Cat. identific. profes. & 17 & $\mathbf{1 8 , 5}$ & 36,3 & 0,9 & \\
\hline Total & 100 & 100 & 34,7 & & \\
(en miles) & 659,1 & 350,4 & & & \\
\hline
\end{tabular}

IRF(1): Cociente de las tasas de feminización de una categoría laboral con respecto a la participación femenina en el empleo total.

IRF(2): Cociente de las tasas de feminización de una categoría laboral con respecto a la participación femenina en el empleo total del subgrupo en el que la categoría está integrada.

FUENTE: Encuesta sociodemográfica (1991, INE) y elaboración propia. 
Como vemos en la tabla 7, las mujeres están escasamente representadas en las tareas de dirección o supervisión. En estos niveles de responsabilidad, el Indice de Represenación Femenina queda muy por debajo de la unidad.

TABLA 7: TRABAJADORES POR CUENTA AJENA, SEGÚN SU RESPONSABILIDAD SOBRE PERSONAL (\%)

\begin{tabular}{|l|c|c|c|c|}
\hline $\begin{array}{l}\text { RESPONSAB. SOBRE } \\
\text { PERSONAL }\end{array}$ & varones & mujeres & t. feminiz. & IRF \\
\hline Direc. supervis. general & 3,6 & 1,1 & 14,8 & 0,4 \\
\hline Super. o dirige cuadros & 2,6 & 1,2 & 19 & 0,5 \\
\hline Super. o dirige empleados & 12,4 & 4,4 & 15,8 & 0,4 \\
\hline Sin responsab. sobre emp. & 79 & 90,9 & 37,9 & 1,1 \\
\hline Situac. no bien definid. & 2,5 & 2,4 & 34,3 & 1,0 \\
\hline $\begin{array}{l}\text { Total } \\
\text { (en miles) }\end{array}$ & 100 & 100 & 34,7 & \\
\hline
\end{tabular}

FUENTE: Encuesta sociodemográfica (1991, INE). Elaboración propia.

\section{LAS CONDICIONES DE TRABAJO: FLEXIBILIDAD E IRREGULARIDAD}

Lo que se ha ido viendo hasta ahora apunta a que las mujeres de la Comunidad Valenciana se han ido conformando como una fuerza de trabajo permanente. El incremento de la ocupación femenina, así como su persistencia entre los desempleados que buscan empleo, a pesar de las escasas oportunidades, apoyan esa hipótesis. Pero estos cambios no han modificado sustancialmente las desigualdades existentes en el mercado laboral entre hombres y mujeres, ni en cuanto a la jerarquización sexual de las posiciones, ni en lo referente a las condiciones de trabajo. Las mujeres siguen soportando una situación más precaria, como nos muestra la mayor flexibilidad e irregularidad en sus relaciones laborales y los menores salarios.

La relación laboral adquiere una forma contractual que puede asumir diferentes modalidades. Estas modalidades van desde los contratos fijos o temporales, a tiempo completo o parcial, con protección o no, y todo ello suele asociarse con otras características del status del empleo, como es el lugar de trabajo, en la propia empresa o en el domicilio, y su caracter formal o informal. 
La información de la que se dispone nos muestra que estas diferencias son muy importantes para el empleo femenino. Las mujeres suelen ser el colectivo, junto a los jóvenes, que mantienen los tipos de contrato más flexibles y los mayores niveles de irregularidad laboral.

\section{a. Contrato de trabajo y flexibilidad}

La temporalidad en el empleo es uno de los indicadores comúnmente utilizados para medir la flexibilidad de la relaciones laborales.

La presencia en nuestro país de esa modalidad de contratación fue relativamente escasa hasta la década de los 80 , pero, a partir de estos años, se ha ido implantando con intensidad. Los cambios introducidos en la reglamentación laboral tuvieron una rápida plasmación en la realidad del mercado de trabajo, y la temporalidad se ha extendido al 31,4\% de los asalariados del país en 1991, según datos de la ESD. En la Comunidad Valenciana se supera esta tasa, elevándose al 35,4.

Tal como muestra la tabla 8 , la temporalidad afecta al $43,5 \%$ de las mujeres que trabajan por cuenta ajena, lo que supone una clara diferencia con los varones sujetos a esta modalidad de contratación, que representan el $31,1 \%$ de los asalariados.

Este tipo de relación laboral no sólo disminuye la capacidad de negociación de los trabajadores, repercute también en las remuneraciones e, indirectamente, dificulta la promoción al fomentar la rotación laboral. Y son, como vemos, las mujeres, junto a los jóvenes de ambos sexos, las que en mayor medida se ven afectadas por este de tipo de contratación sea cual sea su modalidad.

La edad influye claramente en la intensidad de la temporalidad. Como nos muestra la tabla, los menores de 30 años, hombres y mujeres, presentan las mayores tasas de temporalidad, superando el $50 \%$ de los contratos en ambos casos. En este primer segmento, aunque las mujeres presentan una mayor proporción, los valores de las tasas relativas a los dos sexos se acercan bastante, pero, a partir de aquí, se agudizan las diferencias. En el caso de las mujeres, además, con la edad, se incrementa esta modalidad de contratación.

\section{b. El trabajo irregular}

El trabajo irregular, oculto o sumergido, hace referencia a diversas situaciones generalmente caracterizadas por su opacidad: trabajo que no queda reflejado en las estadísticas de empleo, o en la Contabilidad Nacional, trabajo en el que se incumplen las normas laborales sobre protección en el trabajo o protección social. 
TABLA 8: DISTRIBUCIÓN DE LOS TRABAJADORES POR CUENTA AJENA SEGÚN LA CLASE DE CONTRATO (\%).

\begin{tabular}{|l|c|c|}
\hline CLASE DE GONTRATO & varones & mujeres \\
\hline - De duración indefinida & 66,9 & 55,2 \\
- Funcionario público & 6,5 & 10,5 \\
- Fijo continuo & 52 & 36,1 \\
- Fijo discontinuo estacional & 4,7 & 4,8 \\
- Fijo discontinuo sin periodicidad & 3,7 & 3,8 \\
\hline - Temporal o eventual & 31,1 & 43,5 \\
- De aprendiz, formac., práct. & 2,3 & 4 \\
- Estacional o de temporada & 7,4 & 11,3 \\
- De obra o servicio & 2,1 & 3,5 \\
- Eventual & 17,3 & 18,4 \\
- Interino & 0,8 & 2,1 \\
- Otro tipo de relación laboral & 1,3 & 4,2 \\
\hline Total & 100 & 100 \\
(en miles) & 659,1 & 350.4 \\
\hline
\end{tabular}

FUENTE: Encuesta sociodemográfica 1991, INE.

Al hablar aquí de trabajo irregular se hará desde la óptica de la protección social. Se analizan aquellas situaciones en las que la obligación laboral de los empleadores de afiliar a los trabajadores a la Seguridad Social se incumple.

Teniendo en cuenta este indicador, y según la Encuesta Sociodemográfica (INE 1991), en la Comunidad Valenciana el 6,6\% de la población asalariada se encuentra en situación irregular.

De nuevo el género marca diferencias importantes. Son las mujeres las que en mayor medida se encuentran en situación de irregularidad. Tal como podemos ver en la tabla 11, las mujeres representan un tercio del empleo asalariado total (la tasa de feminización es de 32,8\%) y, sin embargo, representan dos tercios del trabajo irregular (la tasa de feminización es del 62,8\%).

Este comportamiento no es igual en todas las ramas de actividad. En «Administraciones Públicas», «Educación» y «Sanidad y Servicios Sociales» la relación entre la tasa de feminización del empleo irregular y la tasa de feminización del empleo total presenta valores casi similares entre hombres y mujeres. Igualmente, habría que añadir que en aquellas ramas de actividad en que las mujeres superan el nivel 
TABLA 9: PROPORCIÓN DE CONTRATOS TEMPORALES, SEGÚN GRUPOS DE EDAD Y SEXO.

\begin{tabular}{|l|c|c|}
\hline GRUPOS DE EDAD & $\begin{array}{c}\text { \% temporal } \\
\text { varones }\end{array}$ & $\begin{array}{c}\text { \% temporal } \\
\text { mujeres }\end{array}$ \\
\hline Menos de 30 años & 52,8 & 56,8 \\
\hline Entre 30 y 39 años & 21,6 & 32,5 \\
\hline Entre 40 y 49 años & 19,3 & 34,5 \\
\hline 50 años y más & 21,6 & 39,3 \\
\hline
\end{tabular}

FUENTE: Encuesta sociodemográfica I991 (INE) y elaboración propia.

TABLA 10: INDICE DE TEMPORALIDAD SEGÚN RAMAS DEACTIVIDAD (\%).

\begin{tabular}{|l|c|c|}
\hline RAMA DE ACTIVIDAD & $\begin{array}{c}\text { Temporalid. femen./ } \\
\text { mujeres asalariadas }\end{array}$ & $\begin{array}{c}\text { Temporalidad/ } \\
\text { total asalariados }\end{array}$ \\
\hline Agricult., ganad., pesca & 78 & 65,5 \\
\hline Industrias extractivas & - & 7,1 \\
\hline Energía e industrias & 45,3 & 31,5 \\
\hline Construcción & 45,4 & 42,6 \\
\hline Comercio & 49,8 & 34,9 \\
\hline Hostelería & 49,1 & 46,9 \\
\hline Transportes y comunicaciones & 34,5 & 13,5 \\
\hline Instituciones financieras & 37,1 & 15,1 \\
\hline Administraciones públicas & 15,9 & 11,1 \\
\hline Educación & 18,2 & 16,1 \\
\hline Sanidad y asistencia social & 32,1 & 28,5 \\
\hline Otros servicios & 44,2 & 38,6 \\
\hline Servicio doméstico & 75,9 & 77,3 \\
\hline Total & 43,5 & 35,4 \\
\hline
\end{tabular}

FUENTE: Encuesta sociodemográfica 1991, INE.

de paridad entre los sexos, como es el caso de las tres citadas más «comercio», «hostelería» y «otros servicios» (tabla 5), esta relación se sitúa por debajo de la media femenina.

El aumento de la flexibilidad y de la irregularidad en la contratación laboral no siempre ha respondido a necesidades del proceso pro- 
TABLA 11: DISTRIBUCIÓN DE LA POBLACIÓN OCUPADA, SEGÚN SU RELACIÓN CON LA SEGURIDAD SOCIAL (\%).

\begin{tabular}{|l|c|c|c|c|}
\hline \multirow{2}{*}{$\begin{array}{l}\text { RELACIÓN CON LA } \\
\text { SEGURIDAD SOCIAL }\end{array}$} & \multicolumn{2}{|c|}{ Comunidad Valenciana } & \multicolumn{2}{|c|}{ España } \\
\cline { 2 - 5 } & varones & mujeres & varones & mujeres \\
\hline Dado de alta & 90,5 & 77,9 & 88,8 & 77,6 \\
\hline No dado de alta & 3,6 & 12,7 & 4,3 & 13,4 \\
\hline Funcionarios & 4,9 & 3,1 & 6,3 & 8,5 \\
\hline No contesta & 1 & 1,2 & 0,6 & 0,4 \\
\hline Total & 100 & 100 & 100 & 100 \\
(en miles) & 886,5 & 432,6 & 8819 & 4310 \\
\hline
\end{tabular}

FUENTE: Encuesta sociodemográfica 1991. INE.

TABLA 12: FEMINIZACIÓN DEL TRABAJO IRREGULAR SEGÚN RAMAS DE ACTIVIDAD.

\begin{tabular}{|l|c|c|c|}
\hline RAMA DE ACTIVIDAD & $\begin{array}{c}\text { T. feminiz. } \\
\text { del empleo }\end{array}$ & $\begin{array}{c}\text { T. feminiz. } \\
\text { emp. irreg. }\end{array}$ & $\begin{array}{c}\text { TF emp. irr } / \\
\text { TF empleo }\end{array}$ \\
\hline Agricult., ganad., pesca & 14,5 & 39,5 & 2,7 \\
\hline Ind. extractivas & - & - & - \\
\hline Energía e industrias & 23,6 & 64,1 & 2,7 \\
\hline Construcción & 2,8 & 13,3 & 4,7 \\
\hline Comercio & 41,9 & 63,8 & 1,5 \\
\hline Hostelería & 39,2 & 69,2 & 1,7 \\
\hline Transportes y comunicaciones & 13,4 & - & - \\
\hline Instituciones financieras & 23,4 & 66,6 & 2,8 \\
\hline Administraciones públicas & 36 & 30,4 & 0,8 \\
\hline Educación & 65 & 64,2 & 0,9 \\
\hline Sanidad y asistencia social & 67,7 & 75 & 1,1 \\
\hline Otros servicios & 45,5 & 68,9 & 1,5 \\
\hline Servicio doméstico & 99,3 & 98,1 & 0,9 \\
\hline Total & 32,8 & 62,8 & 1,9 \\
\hline
\end{tabular}

FUENTE: Encuesta sociodemográfica 1991. INE. 
ductivo. Esta tendencia también se relaciona con la presión para reducir los costes del empleo fijo y asegurar la posibilidad de realizar ajustes futuros en el empleo. La pregunta que podemos hacernos es por qué son precisamente las mujeres las que se ven más afectadas por este fenómeno.

A menudo se argumenta que el mayor rechazo de los hombres a aceptar esas condiciones de trabajo explica el predominio de las mujeres, especialmente casadas, en esta modalidad de contratación. Este argumento coincidiría con el planteamiento de la teoría dual cuando expresa que las mujeres, entre otros grupos, están dispuestas a aceptar este tipo de trabajos porque consideran su relación con el mercado como algo temporal. Esta percepción del trabajo remunerado les permite soportar la incertidumbre, puesto que en cualquier momento pueden volver a sus ocupaciones tradicionales. ${ }^{9}$ Pero también hay que decir que, independientemente de que las mujeres partan de o no de una concepción de su trabajo en el mercado como algo transitorio, lo cierto es que algunas formas de contratación -a tiempo parcial o en el propio domicilio, no es tan claro en el caso del trabajo temporal-permiten una mejor adaptación a las obligaciones que en la esfera reproductiva siguen manteniendo, en la mayoría de los hogares, sólo las mujeres.

\section{CONCLUSIONES}

La consolidación del empleo femenino en este largo período de crisis, no puede considerarse como una evidencia concluyente de que se esté superando la desventaja de las mujeres en el mercado laboral. La relativa protección de la que han disfrutado las mujeres en el mercado de trabajo, que ha supuesto un incremento de la ocupación en los últimos veinte años, a pesar de la crisis de empleo, se explica mejor desde la propia reestructuración sectorial de la economía y de la organización institucional del mercado de trabajo, que desde la disminución de las desigualdades entre hombres y mujeres. Es más, en algunos sectores, ha podido ser precisamente la desventaja relativa de las mujeres la que las ha mantenido en el mercado.

El fuerte proceso de terciarización de la economía que se ha experimentado en el periodo ha beneficiado al empleo femenino. Las mujeres se han refugiado en el sector terciario, el único que ha tenido un comportamiento positivo en la Comunidad Valenciana y en el que una parte importante del empleo ha sido creado en el sector público. Sin

9 Piore, M. «El dualismo como respuesta al cambio y a la incertidumbre», en Toharia, L. (comp.) «El mercado de trabajo: Teorías y aplicaciones». Ed. Alianza, 1983. Madrid. Pgn. 254. 
embargo, su presencia sigue siendo relativamente escasa en el resto de los sectores económicos.

Podemos pensar que la segregación ocupacional ha proporcionado una protección selectiva del empleo femenino. De este modo, aunque la fuerza de trabajo femenina haya actuado como mecanismo amortiguador en alguna rama de actividad considerada individualmente, la mayor presencia en otras que han estado menos afectadas por la crisis ha atenuado esos efectos. Desde esta perspectiva, las características del ciclo son las que han hecho menos vulnerable el empleo femenino.

El resultado es que una parte muy importante de las mujeres ocupadas, a pesar de la crisis, y gracias a la especificidad del ciclo, se han convertido en participantes permanentes de la fuerza de trabajo, lo que, unido a su mayor cualificación previa a la entrada en el mercado de trabajo, pero también posterior, ha modificado sensiblemente su posición, resultando difícil actualmente imaginar que estas mujeres lleguen a ser desplazadas del lugar que ocupan en la estructura ocupacional.

Junto a éstas, las «otras trabajadoras», con o sin experiencia laboral previa, dispuestas a integrarse en el mercado de trabajo, aunque no sea en empleos seguros y bien remunerados, han permanecido durante todo el período, bien como paradas, desanimadas o activas, bien ocupando puestos de trabajo absolutamente precarizados. La mayoría de estas mujeres no cuentan con una cualificación formal y han superado la edad que el mercado suele considerar «normal» para competir en él. Dependiendo de la situación económica familiar están dispuestas a aceptar esos empleos mal remunerados y no reglamentados. Estas mujeres siguen constituyendo una reserva flexible de mano de obra.

Estas características - mano de obra más flexible y menos costosase adaptan perfectamente al tipo de empleo que se ha creado en los últimos años, y explicarían, junto a la tesis de la segregación, la mayor tendencia al alza del empleo femenino en este período.

Nos encontramos pues, por una parte, con que la segregación de la fuerza de trabajo ha llevado a las mujeres a hacerse fuerte en unas ramas de actividad en la que aparecen claramente integradas. Realizan trabajos o desempeñan profesiones que no pueden considerarse propias del mercado secundario, puesto que exigen importantes niveles de cualificación, de experiencia y de respondabilidad.

Ahora bien, aunque a pesar de que en estos sectores las mujeres no actúan como mano de obra secundaria o sustitutoria, es difícil verlas ocupando las posiciones más altas de la jerarquía laboral.

En este sentido se ha avanzado; las mujeres desempeñan, en mayor medida que antes, puestos intermedios, pero, incluso en los sectores ocupacionales feminizados, raramente ocupan los puestos de dirección, aun presentando una cualificación similar a la que presentan los hombres. A menudo se argumenta que esto es consecuencia de la falta 
de preparación de las mujeres y de su falta de dedicación e interés por el trabajo. Opino, sin embargo, con C. Borderías, que «en sectores concretos, los menores niveles de promoción de las mujeres se producen incluso en los casos en que las mujeres tienen idénticos niveles de responsabilidad y de compromiso con el trabajo» ${ }^{10}$.

Por otra parte, hay que destacar que las mujeres siguen siendo un colectivo especialmente sensible a la precarización de sus condiciones de trabajo -temporalidad, irreguralidad, menores salarios-e, indudablemente, la percepción social del papel que hombres y mujeres deben desempeñar en el ámbito del trabajo reproductivo tiene mucho que ver con ello.

La dedicación al trabajo de reproducción, que en muchos casos lleva a considerar el trabajo mercantil como un trabajo secundario y sus retribuciones monetarias como complentarias del salario familiar que representan los ingresos del marido, lleva a que se vea a las mujeres como fuerza de trabajo flexible y menos costosa; las mujeres se convierten en sujetos más adecuados para trabajar en condiciones de irregularidad y temporalidad, mayor rotación y escasa promoción, características todas ellas que responden al mercado secundario.

Un segmento importante de este colectivo femenino, generalmente mujeres casadas con escasa cualificación, están dispuestas a aceptar estas condiciones de trabajo porque, efectivamente, consideran que su trabajo principal se inserta en la esfera de la reproducción, pero, también, en muchos casos, se trata de una adaptación ante las mayores dificultades que encuentran las mujeres para acceder a otro tipo de trabajo. Desde el punto de vista de la demanda, además, se siguen manteniendo vigentes ciertos prejuicios sobre el rendimiento laboral y la posbilidad de que su situación en la familia permita a las mujeres cumplir con sus obligaciones en la esfera mercantil. Estos prejuicios se ven compensados por las menores exigencias en sus condiciones de trabajo, haciéndolas atractivas para ciertos trabajos precarios, pero no para el trabajo en general.

Así, aunque se han producido cambios cualitativos en la participación de la mujer en el mundo del trabajo, no está seguro su progreso mientras se sigan manteniendo los presupuestos socialmente construidos que mantienen la división sexual del trabajo en el hogar y la relacionan con el trabajo en el mercado, reforzando la posición subordinada de las mujeres en él.

Deseo agradecer a A. Alaminos la ayuda prestada en el tratamiento de los datos.

10 Borderías, C. «Las mujeres y el trabajo. Rupturas conceptuales». Ed. Icaria. Barcelona, 1994. Pág. 72. 